\title{
Critical State Behaviour in a Low Dimensional Metal Induced by Strong Magnetic Fields
}

\author{
N. Harrison \\ National High Magnetic Field Laboratory, LANL, MS-E536, Los Alamos, New Mexico 87545 \\ L. Balicas \\ Instituto Venezolano de Investigaciones Cientificas, Apartado 21827, Caracas 1020A, Venezuela \\ J. S. Brooks \\ National High Magnetic Field Laboratory, Florida State University, Tallahassee, Florida 32310 \\ M. Tokumoto \\ Electrotechnical Laboratory, Tsukuba, Ibaraki 305, Japan
}

(October 25, 2018)

\begin{abstract}
We present the results of magnetotransport and magnetic torque measurements on the $\alpha$-(BEDT$\mathrm{TTF})_{2} \mathrm{KHg}(\mathrm{SCN})_{4}$ charge-transfer salt within the high magnetic field phase, in magnetic fields extending to $33 \mathrm{~T}$ and temperatures as low as $27 \mathrm{mK}$. While the high magnetic field phase (at fields greater than $\sim 23 \mathrm{~T}$ ) is expected, on theoretical grounds, to be either to a modulated charge-density wave phase or a charge/spin-density wave hybrid, the resistivity undergoes a dramatic drop below $\sim 3 \mathrm{~K}$ within the high magnetic field phase, falling in an approximately exponential fashion at low temperatures, while the magnetic torque exhibits pronounced hysteresis effects. This hysteresis, which occurs over a broad range of fields, is both strongly temperature-dependent and has several of the behavioural characteristics predicted by critical-state models used to describe the pinning of vortices in type II superconductors in strong magnetic fields. Thus, rather than exhibiting the usual behaviour expected for a density wave ground state, both the transport and the magnetic properties of $\alpha$-(BEDT-TTF $)_{2} \mathrm{KHg}(\mathrm{SCN})_{4}$, at high magnetic fields, closely resemble those of a type II superconductor.
\end{abstract}

\section{INTRODUCTION}

Of all the quasi-two-dimensional (Q2D) organic charge-transfer salts that exist [1], those of the composition $\alpha$-(BEDT-TTF $)_{2} M \mathrm{Hg}(\mathrm{SCN})_{4}$, where BEDTTTF stands for bis(ethylenedithio)tetrathiafulvalene and where $M=\mathrm{K}, \mathrm{Tl}, \mathrm{Rb}$ or $\mathrm{NH}_{4}$, have been, perhaps, the most difficult to understand [2, 3]. While, the $M=\mathrm{NH}_{4}$ salt is a superconductor with a transition temperature $T_{\mathrm{c}} \sim 1 \mathrm{~K}$ 迎, the $M=\mathrm{K}, \mathrm{Tl}$ and $\mathrm{Rb}$ salts all undergo a transition into a more resistive state with a reconstructed Fermi surface below $T_{\mathrm{p}} \sim 8$ (in the $M=\mathrm{K}$ and $\mathrm{Tl}$ salts) or $10 \mathrm{~K}$ (in the $M=\mathrm{Rb}$ salt) [2,3]. The underlying physical reason for the behavioural differences between the isostructural $M=\mathrm{NH}_{4}$ and $M=\mathrm{K}, \mathrm{Tl}$ and $\mathrm{Rb}$ salts remains a contemporary issue. The $M=\mathrm{K}$ salt has, nevertheless, been shown to become superconducting under uniaxial stress applied perpendicular to the conducting layers [5.6], and it has further been suggested that the $M=\mathrm{K}$ and $\mathrm{Rb}$ salts could exhibit filamentary superconductivity even at ambient pressure [7 9].

Unquestionably, the main physical traits of the $M=\mathrm{K}$, $\mathrm{Tl}$ and $\mathrm{Rb}$ salts at ambient pressure, at moderately low temperatures $T \lesssim T_{\mathrm{p}}$ and at magnetic fields $B<B_{\mathrm{k}}$, where $B_{\mathrm{k}}(\approx 23 \mathrm{~T}$ in the $M=\mathrm{K}$ salt $)$ is known as the kink transition field, are more typical of a density wave
(DW) ground state 10,11. Yet, no direct evidence for a superlattice structure has been found. Most of the more recent experimental and theoretical surveys point towards a charge-density wave (CDW) rather than a spindensity wave (SDW) being the more likely candidate [12 [15]. The electrical transport is strongly magnetoresistive, with the increase in resistance below $T_{\mathrm{p}}$ becoming increasingly pronounced in a magnetic field [10, 11, 49. On the approach to $B_{\mathrm{k}}$, however, the magnetoresistance changes, becoming much lower above this field [17]. The nature of the high magnetic field phase $\left(B>B_{\mathrm{k}}\right)$, at low temperatures, has since been the subject of speculation 113,14, 18 22]. The only fully established fact is that the transition field $B_{\mathrm{k}}$ is strongly first order, with hysteresis appearing both in the magnetoresistance and the magnetisation over a few Tesla interval in field.

From the theoretical perspective, $B_{\mathrm{k}}$ lies remarkably close to the critical field $B_{\mathrm{c}}$ at which one would expect a CDW, with a mere transition temperature of $8 \mathrm{~K}$, to approach the Pauli paramagnetic limit 13, 15. A first order transition could be expected, with the material transforming at higher fields either into a normal metal [15], a spatially modulated CDW [13] or a mixed CDWSDW hybrid [23]. The latter, in particular, represents the CDW analogue of the Fulde-Ferrel phase that is anticipated to occur in strongly type II superconducters, 
with suppressed orbital effects, over the equivalent region of the phase diagram 22,25]. Were either of these latter two phases actually to be realised at high magnetic fields, their physical properties have not yet been the subject of a thorough investigation. Experimentally, data has been published that could be considered conducive to the existence of a different thermodynamic phase above $B_{\mathrm{k}}$ at low temperatures 19.26, albeit that the magnetic data, at that time, was discussed largely in the context of SDW's with no metion of CDW's. Yet other data, concerning the unusual beheviour of the interlayer magnetotransport and induced currents in the magnetisation in pulsed magnetic fields, has been notionally connected with the quantum Hall effect (QHE) 20 22. While a definitive experiment has not been performed that can firmly establish either of these latter two scenarios at high magnetic fields $\left(B>B_{\mathrm{k}}\right)$, one cannot dispute the fact that this high magnetic field phase is somewhat exotic.

With a view to understanding more about the high magnetic field phase (at fields above $B_{\mathrm{k}}$ ), in this paper we present the results of extensive measurements of the interlayer magnetotransport and the magnetic torque on several $\alpha$-(BEDT-TTF $)_{2} \mathrm{KHg}(\mathrm{SCN})_{4}$ crystals over a broad range of temperatures, $20 \mathrm{mK}<T<10 \mathrm{~K}$, and in continuous magnetic fields of up to $33 \mathrm{~T}$. The observed behaviour of the magnetotransport and magnetic torque is neither typical of that expected for a DW system, nor does it support recent claims of the QHE in this material. For example; as the sample is cooled in a magnetic field, the resistivity undegoes an abrupt drop at $\sim 3 \mathrm{~K}$, at all fields $B \gtrsim B_{\mathrm{k}}$, with the drop being particularly pronounced at integer Landau level filling factors, falling exponentially by roughly two orders of magnitude. The occurrence of a change in slope in the magnetic torque at precisely the same temperature, on field-cooling the sample, lends further support to the existence of a different low temperature thermodynamic phase below $T_{\mathrm{c}} \lesssim 3 \mathrm{~K}$ [19]. At lower temperatures still, (i.e. $T \lesssim 2 \mathrm{~K}$ ), the field-dependence of the magnetic torque develops a pronounced hysteresis at all fields above $B_{\mathrm{k}}$ that increases approximately exponentially with decreasing $T$, but with the hysteresis being particularly strong at half integer Landau level filling factors. Only at much lower temperatures $(T \sim 27 \mathrm{mK})$ does the hysteresis become more pronounced at integer filling factors [20]. While the hysteresis throughout the entire high magnetic field phase is difficult to explain in terms of conventional DW ground states or the QHE, it has many of the features of a critical state model like those used to explain magnetic hysteresis in type II superconductors. In the absence of any other model specific to the high magnetic field phase of $\alpha$-(BEDT-TTF $)_{2} \mathrm{KHg}(\mathrm{SCN})_{4}$, the irreversible hysteretis, together with the sharp drop in the resistivity at low temperatures, is remarkably similar to the behaviour of superconductor in strong magnetic fields. Such behaviour cannot be explained consistently in terms of the QHE or any conventional DW ground state.

\section{EXPERIMENTAL}

The samples chosen for this study, referred to hereon as 1, 2 and 3, were grown using conventional electrochemical means [27], while static magnetic fields extending to $\sim 33 \mathrm{~T}$ were provided by the the National High Magnetic Field Laboratory, Tallahassee. Temperatures between $\sim 20 \mathrm{mK}$ and $\sim 1.6 \mathrm{~K}$ were provided by a top-loading dilution refrigerator, with higher temperatures obtained using a ${ }^{3} \mathrm{He}$ refrigerator. Magnetotransport measurements were made using standard four wire techniques with low frequency $(\sim 10 \mathrm{~Hz})$ currents of between 1 and $10 \mu \mathrm{A}$ applied perpendicular to the conducting layers. For the magnetic torque measurements, the samples were mounted on the moving plate of a phosphor bronze capacitance cantilever torque magnetometer, which was itself attached to a rigid but rotatable platform in such a way that the axes of torque and rotation were parallel to each other, but perpendicular to the applied magnetic field $B$. The angle between $B$ and the normal to the capacitance plates was approximately the same as the angle $\theta$ between $B$ and the normal to the conducting planes of the sample. The capacitance of $\sim 1.3 \mathrm{pF}$ was measured by means of a capacitance bridge energized at $5 \mathrm{KHz}$ and $30 \mathrm{~V}$ (rms); the largest change in $\mathrm{C}$ observed throughout the experiment at the highest magnetic field was $\sim 0.04 \mathrm{pF}$, corresponding to a net angular displacement of $\sim 0.1^{\circ}$. Torque interaction effects at $\theta=7^{\circ}$ were therefore not a significant factor. To eliminate possible artefacts due to time constants of the instrumentation setup or the data acquisition system, the capacitance was measured with the time constant of the lock-in amplifier set to a low value of $10 \mathrm{~ms}$. Furthermore, both the output of the lock-in amplifier and the shunt voltage that determines the current flowing in the Bitter magnet coils, were measured using a digitizer with resolution of $\sim 50 \mu \mathrm{s}$, while the field was swept slowly at $\sim 8 \mathrm{mTs}^{-1}$.

\section{TEMPERATURE-DEPENDENCE OF THE MAGNETORESISTANCE}

Examples of the interlayer magnetoresistance measured in $\alpha$-(BEDT-TTF $)_{2} \mathrm{KHg}(\mathrm{SCN})_{4}$ sample 1 at selected temperatures are shown in Fig. 11. The magnetoresistance displays the usual behaviour observed for this material, reaching a maximum at $\sim 10 \mathrm{~T}$ before falling again in a linear fashion as the kink transition field is approached [10,27]. In accordance with these earlier studies, the oscillations within the low magnetic field DW phase exhibit a pronounced second harmonic. Above 
the kink transition, at temperatures higher than $\sim 3 \mathrm{~K}$, the oscillations grow rapidly in amplitude with increasing field. The behaviour of the interlayer Shubnikov-de Haas $(\mathrm{SdH})$ waveform over this range of temperatures is well understood in these materials 28,29. At integer Landau level filling factors, when the chemical potential $\mu$ is situated in a Landau gap, the $\mathrm{SdH}$ maxima increase with decreasing temperature in a insulating-like fashion. Conversely, at half-integer filling factors, the resistivity of the minima behave in a metallic fashion. This is entirely consistent with the theoretically expected behaviour of a quasi-two-dimensional metal in a magnetic field [28,29.

What is not predicted by the simple theory is the abrupt inversion of the waveform at low temperatures, resulting in the high temperature $(T \gtrsim 3 \mathrm{~K}) \mathrm{SdH}$ maxima becoming minima at low temperatures $(T \lesssim 3 \mathrm{~K})$ with a resistivity several times lower than that of the sample at zero field. Figure 1] does not constitute the first observation of this effect; previous studies had reported this effect in both continuous and pulsed magnetic fields [21,22]. Because no phase inversion is observed in the dHvA effect 21,22, which is entirely a thermodynamic function of state, this implies that the Landau level structure remains largely unchanged over the same temperature range. It was the occurrence of this phase inversion only in the magnetotransport that was then attributed to the effect of a chiral Fermi liquid [21,22], following a suggestion that the QHE may be taking place at high magnetic fields in this material [20]. Because the interlayer resistance of the bulk of the sample becomes insulator-like at integer filling factors according to magnetotransport theory [28,29, higher conductivity chiral Fermi liquid states were proposed to take over the majority of the interlayer conductance. For this conjecture to explain the most recent data (i.e. Fig. 1), the interlayer conductivity of the edge states, which occupy $\sim 1$ part in $10^{4}$ of the sample cross section, would have to be both strongly temperature-dependent and attain a conductivity at least $10^{6}$ times higher than that of the field-averaged (or background) conductivity of the bulk.

Since this conjecture was made, the interlayer conductance of chiral surface states has been measured directly in semiconductor superlattices that at the same time quite clearly exhibit the QHE [30,31. Not only is the conductance of these states found to be temperatureindependent, as predicted by chiral Fermi liquid theory 32, 33, but their net conductance is also observed not to be particularly high, resulting in only a weak suppression of the SdH maxima. This behaviour is therefore quite different from that observed in $\alpha$-(BEDT$\mathrm{TTF})_{2} \mathrm{KHg}(\mathrm{SCN})_{4}$. When coupled with the fact that quantized Hall plateaux proportional to $\hbar / i e^{2}$ have not been observed 49 (in the $M=\mathrm{Tl}$ salt), the chiral Fermi liquid model can no longer be considered valid.

A more distinct behaviour emerges when the resistivity, both at integer and half-integer filling factors, is plotted versus temperature in Fig. 2. For two different samples, the resistivity varies weakly with temperature for $T \gtrsim 3 \mathrm{~K}$, but then undergoes an abrupt drop for $T \lesssim 3 \mathrm{~K}$. This drop is particularly pronounced at integer filling factors (i.e. when $\mu$ is situated in the Landau gap of the Q2D pockets), but is also clearly discernable at half-integer filling factors (i.e. when $\mu$ is situated in the middle of a Landau level). This behaviour is somewhat similar to that observed by Kartsovnik et al. [19], except that in the present case, at integer filling factors in sample 1 , the resistivity is observed to fall by two orders of magnitude between $3 \mathrm{~K}$ and $490 \mathrm{mK}$, reaching $1.6 \Omega$ at the lowest temperature. While $1.6 \Omega$ does not sound that low, it is $\sim 2000$ times lower than the room temperature resistance of $3.6 \mathrm{k} \Omega$ for this sample, and this is even in the presence of $\mathrm{a} \sim 30 \mathrm{~T}$ magnetic field. In spite of the fact that the samples were cooled in a dilution refrigerator on a subsequent experimental run to temperatures as low as $20 \mathrm{mK}$, zero resistivity was never observed. The abrupt change in resistivity versus temperature $\rho(T)$ has previously been interpreted as evidence for a phase transition [19], and the present study appears to support this claim. An abrupt drop in the resistance, as observed here, cannot be explained by chiral Fermi liquid theory 32,33, nor is the occurrence of a transition at half-integer filling factors (as well as integer filling factors) compatible with the QHE. Thus, it would appear to be the case that the inversion of the $\mathrm{SdH}$ waveform is merely an artefact of the drop in resistivity being more pronounced at integer filling factors.

From a phenomenalogical point of view, the low temperature interlayer resistivity in Fig. 2 obeys an approximate $\rho \propto \exp \left(T / T_{0}\right)$ law, with $T_{0} \sim 520 \mathrm{mK}$ at integer filling factors for both samples 1 and 2 . Such an exponential law is clearly unlike that of a normal metal, and the very observation of a sudden drop in resistivity is not expected for a DW ground state. Transitions into DW ground states are invariably followed by an immediate increase in resistivity on decreasing the temperature through the transition 34, owing to a net loss of carriers. A drop in resistivity, of the form observed here, more commonly precedes a superconducting state. One could argue that, while a zero resistivity state is not observed, zero resistance states are always more difficult to come by in strong magnetic fields owing to vortex dynamics [35.36. It is also interesting to note that the exponential dependence of the resistivity at low temperatures is strikingly similar to that observed in the filamentary superconductor $\mathrm{La}_{2} \mathrm{CuO}_{4-y}$ [37.

\section{TEMPERATURE-DEPENDENCE OF THE MAGNETIC TORQUE}

The temperature-dependence of the magnetotransport alone cannot be considered as proof for a transition into 
a new thermodynamic phase. This usually requires measurement of a thermodynamic function of state, such as specific heat or magnetisation. Specific heat measurements have thus far confirmed the existence of a second order transition into a low temperature phase within the low magnetic field regime $\left(B<B_{\mathrm{k}}\right)$ [38]. Within this same phase, magnetic moment measurements, made using a superconducting quantum interference device (SQUID) in fields of $5 \mathrm{~T}$, have shown that the susceptibility drops most notably when the field is oriented within the conducting planes [39]. Such a behaviour could be expected either for a CDW or a SDW phase following a net loss in Pauli paramagnetism with the opening of gaps on the Fermi surface [34. Only a very weak change, however, was observed when the magnetic field was oriented perpendicular to the conducting layers. Sasaki et al. notionally connected this anisotropy with antiferromagnetism associated with a SDW ground state [39], although there has since not been any compelling evidence supporting this hypothesis 12.40.

An alternative, yet rather trivial, explanation could be that when the field is oriented perpendicular to the conducting layers, the loss in Pauli paramagnetism is balanced by a loss in Landau diamagnetism following the partial opening of gaps on the Q2D Fermi surface pockets due to a CDW. Gaps on the Q2D pocket are, after all, predicted by all models of the reconstructed Fermi surface 41]. Perhaps the strongest evidence for the latter explanation is that the magnetic susceptibility is completely isotropic for fields oriented within the planes, as demonstrated by the magnetic torque measurements of Christ et al. [42]. This detail would be difficult to explain in terms of antiferromagnetism, but is quite easy to explain in terms of Landau diamagnetism, which in a Q2D conductor, manifests itself only perpendicular to the conducting layers.

The essential advantage of magnetic torque measurements is that they are sensitive only to the magnetic anisotropy, with the net torque being given by the product

$$
\tau=\mathbf{M} \times \mathbf{B}
$$

Thus, if we consider the susceptibility to be resolved into its components perpendicular to the conducting layers $\chi_{\perp} \equiv M_{\perp} / B$ and parallel to the conducting layers $\chi_{\|} \equiv$ $M_{\|} / B$, the net magnetic torque is then given by

$$
\tau=\frac{1}{2}\left[\chi_{\|}-\chi_{\perp}\right] B^{2} \sin 2 \theta,
$$

where $\theta$ is the angle between the magnetic field and the normal to the conducting layers (i.e. the $\mathbf{b}$ axis of the crystal). With the first term of Equation (2) being dominant and negative, according to Sasaki et al. 39], the change in anisotropy on entering the low temperature DW phase exerts a net negative torque on the sample, that attempts to align the $\mathbf{b}$ axis of the sample more closely with $B$. This is exactly the signature observed by Christ et al. 42]. For a constant anisotropy $\chi_{\|}-\chi_{\perp}$, the negative torque should increase in a manner that is proportional to $B^{2}$. The fact that this is not the case, as shown originally by Christ et al. [43] and again in Fig. 3, could be considered as further supportive evidence for our explanation in terms of Landau diamagnetism, whereby the Landau diamagnetic contribution from the Q2D pocket returns gradually at higher magnetic fields due to magnetic breakdown across the CDW gaps.

Even though the anisotropy becomes reduced at high magnetic fields, a change in magnetic torque on fieldcooling the sample is still visible in Fig 4 . This is perhaps assisted by the increasing sensitivity $\left(\propto B^{2}\right)$ of torque magnetometry with field. While the reason for the continued anisotropy at high magnetic fields is not obvious, the abrupt change in slope in fields as high as $\sim 30.1 \mathrm{~T}$ clearly indicates the continuing presence of a thermodynamic phase boundary. The field of $30.1 \mathrm{~T}$ was chosen because, at this field, $\mu$ is exactly that for a half-integer filling factor, thereby eliminating any possible contribution to the torque from the dHvA signal [19. If our interpretation of the magnetic measurements of Christ et al. and Sasaki et al. is correct, this could implies that the loss of Landau diamagnetism on entering the low temperature, high magnetic field phase continues to be the dominant factor. Were part of the sample to become superconducting at high magnetic fields, as might be suggested from the behaviour of the resistivity, it is unclear how this would affect the results. Usually, the pure Abrikosov diamagnetism of the vortex lattice of a Q2D superconductor is largest when the field is applied perpendicular to the conducting layers [35]. The torque resulting from the net gain in Abrikosov diamagnetism should therefore have the opposite sign of that coming from the loss in Landau diamagnetism. For our results to be consistent with the establishment of a high magnetic field-induced superconducting state, the Landau diamagnetism would have to be larger in magnitude than the Abrikosov diamagnetism at these fields. Whether this is the case depends on parameters which are presently unknown.

\section{HYSTERESIS IN THE MAGNETIC TORQUE}

One of the most intriquing apsects of this material at high magnetic fields is its magnetic hysteresis. An example of the magnetic torque of sample 3 measured both on rising and falling magnetic fields at $27 \mathrm{mK}$ is shown in Fig. 5. Apart from the fact that the temperature is lower in the current work, the hysteresis is very similar to that observed by Christ et al. 44], although this effect was largely disregarded as originating from a "complex magnetic groundstate," with the original interpretation 
of the anisotropic susceptibility work of Sasaki et al. [39] in terms of antiferromagnetism, essentially forming the foundation of their argument.

Consideration of the fact that only the anisotropy of the magnetic suscptibility gives rise to magnetic torque is the key to understanding the behaviour of the magnetic torque in this material. Hence, because the $\mathrm{dHvA}$ effect is the oscillatory component of the Landau diamagnetism, thereby only involving orbital effects within the conducting planes, it manifests itself as large oscillations of the magnetic torque that are rather straightforward to interpret. At low magnetic fields $\left(B<B_{\mathrm{k}}\right)$, the oscillations exhibit the usual double-peaked structure that has been interpreted both as spin-splitting [10,27,45, 48] and as the frequency-doubling effect that accompanies a pinned CDW or SDW phase [15]. This is well known to be an effect observed at small angles $\left(\theta \lesssim 20^{\circ}\right)$ in this material (as well as the $M=\mathrm{Tl}$ and Rb salts), which occurs only within the low magnetic field DW phase. On increasing the field at these low temperatures (i.e. $T=27 \mathrm{mK}$ ), the kink transition is observed to be extremely abrupt, with a small but reproducible spike feature signalling the transformation into the high magnetic field regime at $B \sim 24.2 \mathrm{~T}$ in sample 3 . Above this field, the dHvA oscillations develop the characteristic triangular form that has been observed within the high magnetic field phase 43 45.

The sudden change in the waveform that occurs at $\sim 24.2 \mathrm{~T}$ on the rising magnetic field and at $\sim 21.4 \mathrm{~T}$ on the falling magnetic field is indicative of a first order phase transition between two distinctly different regimes. Because the magnetic torque is entirely reversible within the low magnetic field DW phase, provided the field does not exceed $\sim 24.2 \mathrm{~T}$, both the dHvA oscillations and the monotonic background torque remain unchanged when the direction of sweep of the magnetic field is reversed. Similarly, when the field sweep direction is reversed within the high magnetic field phase, the $\mathrm{dHvA}$ oscillations continue to have the same triangular form, provided that the field is not swept below $\sim 21.4 \mathrm{~T}$. This type of behaviour indicates that the low and high magnetic field phases are immiscible, with the formation of domains being energetically unfavourable. Because it costs energy to mix the two phases, the low magnetic field DW phase is "supercooled" on increasing the magnetic field until the free energy difference between the two phases is no longer sustainable. To help understand this behaviour in $\alpha$-(BEDT-TTF $)_{2} \mathrm{KHg}(\mathrm{SCN})_{4}$, it is instructive to consider a simplified model where the zero temperature free energies of the low and high magnetic field regimes are approximated as

$$
F_{0} \approx-g_{1 \mathrm{D}}\left[\frac{\Delta_{0}^{2}}{2}-h^{2}\right]
$$

and

$$
F_{x} \approx-g_{1 \mathrm{D}} \frac{\Delta_{x}^{2}}{2}
$$

respectively, with $g_{1 \mathrm{D}}$ being the density of Q1D states and $h=g \mu_{\mathrm{B}} B / 2$ [13,15,23], and we assume, to first order, that the high magnetic field phase is not affected by magnetic field. Here, the subscript 0 denotes the CDW phase that is stable at low magnetic fields, while $x$ denotes the spatially modulated or CDW-SDW hybrid phase [13,23]. If we then assume the free energy of the domain term $F_{\text {mix }} \approx \epsilon y(1-y)$ to be approximately parabolic (i.e. the lowest order even funtion of $y$ ), with $y$ being the fraction of the material in the low magnetic field phase, then no domain structure can ever be stable provided $\epsilon \geq 0$. Since the total free energy is

$$
F_{\text {tot }} \approx y F_{0}+(1-y) F_{x}+\epsilon y(1-y),
$$

it then follows that, on increasing the magnetic field within the low magnetic field regime, within which $y=1$, the material cannot "snap" into the high magnetic field phase until $\partial F_{\text {tot }} / \partial B \lesssim 0$. Conversely, on decreasing the magnetic field from within the high magnetic field regime, within which $y=0$, the material cannot snap into the low magnetic field phase until $\partial F_{\text {tot }} / \partial B \gtrsim 0$. The kink transitions for rising and falling magnetic field are therefore

$$
h_{\mathrm{k}}=\sqrt{\frac{\Delta_{0}^{2}-\Delta_{x}^{2}}{2} \pm \frac{\epsilon}{g_{1 \mathrm{D}}}},
$$

with the \pm sign corresponding to the direction of sweep of the magnetic field. The abruptness of the transitions should be accompanied by the release of latent heat, although this cannot be detected in the present isothermal experiment. If we assume the strength of the coupling to be similar in the two ordered regimes, then the ratio of order parameters $\Delta_{x} / \Delta_{0}$ is equal to the ratio of transition temperatures $T_{\mathrm{c}} / T_{\mathrm{p}} \sim 3 / 8$, and we obtain $\Delta_{0} \sim 4.1 \mathrm{meV}$ and $\Delta_{x} \sim 1.5 \mathrm{meV}$ on inserting $B_{\mathrm{c}} \equiv B_{\mathrm{k}} \sim 23 \mathrm{~T}$. Meanwhile, for $1 / g_{1 \mathrm{D}} \sim 30 \mathrm{meV}$, we obtain $\epsilon \sim 24 \mu \mathrm{eV}$.

The hysteresis that starts at magnetic fields above $\sim 24.5$ has quite a different form from that associated with the kink transition. Since (1) the dHvA oscillations have the same shape and size between rising and falling magnetic fields and (2) the hysteresis takes place continuously over a wide interval in field (from $\sim 24.5 \mathrm{~T}$ up to the highest available field of $\sim 33 \mathrm{~T}$ ), this new type of hysteresis appears not to be a consequence of a second prolonged first order phase transition as a function of magnetic field, as was suggested by McKenzie [13]. For a first order phase transition to take place over such an extended interval of field, either (1) the free energies of the two competing phases would have to be very similar but with slightly different dependences on $B$ or $\mu$, or (2) the mixing term $\epsilon$ would have to be very large (and negative). Were this the case, the hysteresis would 
have to result from two competing CDW or CDW-SDW hybrid phases with different anisotropic magnetic susceptibilities, different nesting vectors and therefore different reconstructed Fermi surface topologies. Any difference in Fermi surface topology between rising and falling magnetic field should ultimately manifest itself on the dHvA oscillations. This is to be expected since the peak-topeak amplitude of the dHvA waveform is directly proportional to the number of Q2D states, while the gradient on the falling side of the oscillation (i.e. at integer filling) is proportional to the density of background states [28]. As it turns out, however, there is no detectable change in the peak-to-peak height nor in the falling slope of the $\mathrm{dHvA}$ oscillations between rising and falling fields in Fig. 5 , at least for $B \gtrsim 26 \mathrm{~T}$. In fact, from the fraction of the waveform $\gamma=g_{2 \mathrm{D}} /\left(g_{2 \mathrm{D}}+g_{1 \mathrm{D}}\right)$ over which the dHvA magnetisation increases with field [28], it is rather straight forward to infer that the density of background states is $68 \pm 5 \%$ that of the Q2D pocket, both on rising and falling fields, therefore having the same density of states, within experimental uncertaintly, as the Q1D Fermi surface sheets prior to nesting 411. This could imply that the Q1D states are not nested (or "ungapped"). Indeed, it has been a common conclusion of all quantum oscillation and angle-dependent magnetoresistance oscillation measurements, that the Fermi surface appears to be unreconstructed within the high magnetic field phase [10,18,28]. Alternatively, the high magnetic field phase could be a superconductor, whereby (in the absence of a $\mathbf{Q}$-vector) the gap automatically remains pinned to the oscillating chemical potential, enabling the Q1D Fermi surface sheet to continue affecting the waveform of the dHvA oscillations. One could also envisage a scenario whereby the $\mathbf{Q}$ vectors of a CDW or CDW/SDW phase adjust themselves continuously in order to maintain the gap symmetrical with respect to the oscillating chemical potential; this situation would therefore contrast from that in the low magnetic field phase of $\alpha$-(BEDT-TTF $)_{2} \mathrm{KHg}(\mathrm{SCN})_{4}$ 15] and that in $\mathrm{NbSe}_{3}$ [49]. In either case, the size and shape of the quantum oscillations indicates that there is no discernable difference in the density of states between rising and falling magnetic fields, therefore making it somewhat unlikely that the electronic ground states could be different between rising and falling magnetic fields. The fact that no hysteresis is observed in the magnetotransport further implies that it cannot be the electronic structure which is itself hysteretic.

The only other possibility is that the hysteresis originates from some form of dynamic magnetism involving the ferromagnetic alignment of spins or circulating currents. Whenever magnetic hysteresis occurs, whether it originates from ferromagnetism, metamagnetism, vortex pinning in a type II superconductor or even induced currents in a quantum Hall system, the tendency is always to retain magnetic flux within the sample. Using the field- cooled magnetisation as a point of reference (or the magnetisation averaged between up and down sweeps), the magnetisation is always slightly more diamagnetic on the rising field and slightly more paramagnetic on the falling field. Only the anisotropy of the magnetic susceptibility gives rise to magnetic torque: the sign of the hysteresis observed here thus implies that it involves magnetic moments that are predominantly oriented perperdicular to the conducting planes, or equivalently, currents flowing within the conducting planes. Clearly, $\alpha$-(BEDT$\mathrm{TTF})_{2} \mathrm{KHg}(\mathrm{SCN})_{4}$ contains no magnetic ions with partially filled $d$ - or $f$-electron shells, so we can eliminate this rather trivial source of ferromagnetism or metamagnetism. The hysteresis in Fig. 5 is therefore more easy to explain in terms of induced currents. The fact that the hysteresis is observed only in the magnetic torque and not in the magnetotransport could be considered consistent with this hypothesis. These cannot be quantum Hall currents, however, since the hysteresis is observed at all fields $B \gtrsim 24.5 \mathrm{~T}$, both at integer and half-integer filling factors.

\section{IRREVERSIBLE PROCESSES}

The behaviour that is probably most supportive of the notion of induced persistent currents, is the irreversibility of the magnetic torque on stopping or reversing the sweep direction of the magnetic field. In most metals, including those with DW phases, the magnetisation is usually reversible, as is the case, for example, within the low magnetic field DW phase (i.e. see Fig. 5). On sweeping the field back and forth within the high magnetic field phase, on the other hand, even over a very small interval in field, the magnetic torque of $\alpha$-(BEDT-TTF $)_{2} \mathrm{KHg}(\mathrm{SCN})_{4}$ is observed to be entirely irreversible, giving rise to a hysteresis loop of the form shown in Fig. 6(a). In fact, the nature of the hysteresis has several of the characteristic features of a critical state model, such as the Bean model [35, 50] that is used to describe magnetic hysteresis caused by vortex pinning in type II superconductors. This becomes particularly evident in Fig. 6(b) when the monotonic background and $\mathrm{dHvA}$ contributions are subtracted; the background is obtained by averaging data taken on full up and down sweeps of the magnetic field. In Fig. 6(b) we can see that, on reversing the sweep direction of the magnetic field, the magnetic hysteresis increases gradually until a critical value is reached. In type II superconductors, this would be proportional to the critical current density $j_{\mathrm{c}}$. In Fig. E(b), the induced magnetisation corresponding to the critical state is that obtained by performing full up and down sweeps [also shown in Fig. [6(b)]. By "critical state" we imply that the sample has a tendancy to trap flux exactly like a type II superconductor, with the local currents determining the local magnetic field gradient. While the unit 
of trapped flux in type II superconductors is the vortex which contains a net flux equivalent to that of the flux quantum, it is unclear what the equivalent unit of trapped flux is in the high magnetic field phase of $\alpha$ $(\mathrm{BEDT}-\mathrm{TTF})_{2} \mathrm{KHg}(\mathrm{SCN})_{4}$.

It nevertheless follows from the critical state model as normally applied to type II superconductors, that as soon as the direction of sweep of the magnetic field is reversed, the initial slope of the magnetisation with respect to magnetic field is given by

$$
\chi=\frac{\partial M}{\partial B}=-\frac{f[1-\eta]}{\mu_{0}},
$$

where $-1 / \mu_{0}$ corresponds to perfect diamagnetism, $\eta$ is the demagnetisation factor and $f$ is the volume fraction of the sample in which pinning occurs. This would then be, perhaps, as close to performing a Meissner-type experiment as one could get in extreme magnetic fields. If we consider that the anisotropic hysteresis originates from currents flowing only within the conducting planes and that $\eta \sim 0.5$ (as for a cylinder), upon taking the initial slope of the magnetic torque in Fig. 6(b), we obtain $f \sim 1 \%$ for sample 3 (of volume $\sim 0.8 \mathrm{~mm}^{3}$ ). Thus, were the hysteresis in Fig. 6 really caused by vortex pinning, this would imply either that only $1 \%$ of the vortices are pinned, or that only $1 \%$ of the sample is superconducting. This conclusion, that only part if the sample is pinned or superconducting, could be considered consistent with the failure of the sample to transform into a bulk zero resistance state. The value of $f \sim 1 \%$ would, of course, be underestimated were any of the supercurrent to flow perpendicular to the conducting planes via Josephson tunneling. The value of $1 \%$ should therefore be considered as a lower limit. That it is necessary to sweep the magnetic field by $\sim 0.4 \mathrm{~T}$ before the trapped flux in the sample is completely reversed, could either imply that the local field difference in parts of the sample reaches strengths of order $0.4 \mathrm{~T}$, or that shape effects, which give rise to demagnetisation, are important.

Another property of the critical state model as applied to type II superconductors, is the that they often give rise to relaxation phenomena, through thermally assisted flux flow or quantum flux creep [35, 36, 51]. One way to test whether such effects occur in $\alpha$-(BEDT$\mathrm{TTF})_{2} \mathrm{KHg}(\mathrm{SCN})_{4}$ is to monitor the dependence of the width of the hysteresis loop on sweep rate. Non-linear current-voltage characteristics have already been detected in pulsed magnetic fields experiments, although it is quite likely, should $\alpha$-(BEDT-TTF $)_{2} \mathrm{KHg}(\mathrm{SCN})_{4}$ (or the $M=\mathrm{Tl}$ salt) be a superconductor, that these experiments were performed close to the flux flow regime [20,52. On sweeping the field at rates between $8 \mathrm{mTs}^{-1}$ and $50 \mathrm{mTs}^{-1}$ in Fig. 6, the hysteresis increases by no more than $10 \%$, implying that the relaxation rate is rather low. A more notable degree of relaxation of the magnetic torque is observed by stopping the sweep of the magnetic field abruptly and then observing changes over several minutes. At integer filling factors [Fig. 17(a)], the magnetic torque decays much more rapidly than at halfinteger filling factors [Fig. If(b)] and is considerably more noisy. On fitting the Anderson-Kim flux-creep model 35] (making the substitution of $\tau$ for $M$ )

$$
\tau_{\text {in }} \approx \tau_{\text {crit }}\left[1-\frac{\alpha_{1}}{2 \alpha_{\mathrm{c}}} \ln t\right],
$$

which is thought to be applicable at low temperatures in most type II supeconductors, the better fit is obtained at half-integer filling factors, with the characteristic parameter $\alpha_{1} / \alpha_{\mathrm{c}}$ for logarithmic decay being of order $1 / 620$. This is certainly within an order of magnitude of the theoretical prediction for most type II superconductors [35.

\section{FIELD AND \\ TEMPERATURE-DEPENDENCE OF THE HYSTERESIS}

Perhaps, a further analogy can be made with type II superconductors on consideration of the temperature dependence of the width of the hysteresis loop. In many type II superconductors, this is found to be strongly dependent on temperature, caused, for example, by the dependence of the vortex pinning potential $U_{\mathrm{c}}(T)$ on $T$ [36]. Such a behaviour is also observable for the critical state of $\alpha$-(BEDT-TTF $)_{2} \mathrm{KHg}(\mathrm{SCN})_{4}$, as shown in Fig. B(a), albeit the hysteresis contains an oscillatory component. While no hysteresis could be detected at $T \sim 4 \mathrm{~K}$, it is already quite pronounced by $1.16 \mathrm{~K}$, particularly at halfinteger filling factors. Half-integer filling factors, realised whenever $F / B+1 / 2$ assumes an integer value, are depicted in Fig. 8 by solid vertical lines. Note that the oscillatory component of the width of the magnetic hysteresis loop oscillates in quadrature with the dHvA oscillations [extracted in Fig. 8(b)], but in phase with the density of states, with the hysteresis and the density of states at $\mu$ both exhibiting maxima at half-integer filling factors. Clearly, as discussed in the preceding section, it cannot be the dHvA oscillations themselves that are hysteretic because (1) the dHvA and oscillatory component of the hysteresis effects are at quadrature with respect to each other and (2) the actual dHvA amplitude is only relatively weakly dependent on temperature over the same range $27 \mathrm{mK}<T<1.15 \mathrm{~K}$, shown in Fig. 8(b). This together with the absence of any hysteresis in the $\mathrm{SdH}$ waveform, again, is consistent with our hypothesis in terms of induced currents.

As $T \rightarrow 0$, the hysteresis becomes double-peaked, with the second sharper and more strongly temperaturedependent peak emerging at integer filling factors. Sharp peaks in the hysteresis were observed at integer filling 
factors in pulsed magnetic fields [2022], and it was this "coincidence" that led to the data being interpreted in terms of the QHE. When plotted as a function of temperature in Fig. 9, the hysteresis is strongly dependent on temperature both at integer and half-integer filling factors, with the form of the $T$-dependence not being too different from that typically observed in high temperature superconductors [36].

\section{PHASE DIAGRAM}

It is instructive to construct a tentative phase diagram for $\alpha$-(BEDT-TTF $)_{2} \mathrm{KHg}(\mathrm{SCN})_{4}$, from the data accumulated, at least for fields applied within $\theta \sim 10^{\circ}$ of the $\mathbf{b}$ axis of the crystals. Solid symbols have been chosen for the thermodynamic data, in Fig. 10, which is often the most reliable indicator for a thermodynamic phase transition. Solid squares denote the change in slope of the field-cooled magnetic torque extracted from Fig. 16. At high magnetic fields, this agrees well with the resistive transition at low temperatures (indicated by open circles), as, for example, can be seen in both samples 1 and 2 in Fig. 2. Note that this transition is not sampledependent; only the extent to which the resistivity decreases at low temperatures is sample-dependent, varying somewhat between samples 1 and 2 .

At low magnetic fields, the field-cooled magnetic torque data agrees rather well with the transition into the low magnetic field DW phase obtained from specific heat measurements [38], represented here by solid triangles. In the vicinity of the kink transition, however, the second order phase boundary, on cooling, becomes more difficult to extract from resistivity data, owing to the competition between different types of resistivity behaviour from the low magnetic field DW and exotic high magnetic field phases. For this reason, these points, depicted here as open squares, have been taken from the non-hysteretic kink observed in the magnetoresistance at higher temperatures between $2.5 \mathrm{~K}$ and $5 \mathrm{~K}$. The hysteretic kink transition at lower temperatures, on the other hand, has been extracted from the magnetic torque measurements made in the dilution refrigerator, denoted here by vertical crosses on the rising magnetic field and diagonal crosses on the falling magnetic field.

The emerging phase diagram can be described as follows: at temperatures above $\sim 8 \mathrm{~K}, \alpha$-(BEDT$\mathrm{TTF})_{2} \mathrm{KHg}(\mathrm{SCN})_{4}$ behaves like any other organic metal, transforming into a DW phase at low temperatures. Given the lack of evidence for static antiferromagnetically configured spins [12,40 and the close similarity of our experimentally determined phase diagram to that predicted both by McKenzie [13] and Zanchi et al. [23, this is more likely to be the $\mathrm{CDW}_{0}$ phase. Certainly, the first order transition into a new low temperature, high magnetic phase is mostly consistent with the theoretical phase diagram proposed by McKenzie [13]. What is particularly interesting in the current work is that while the high magnetic field phase $\left(\mathrm{CDW}_{x}\right)$ is expected, largely on theoretical grounds, to be a modulated CDW phase or mixed CDW-SDW hybrid, its transport and magnetic properties are quite unlike those observed in all known DW systems [34]. Rather, its physical properties more closely mimic those of a type II superconducting phase in strong magnetic fields.

\section{CONCLUSION}

We have shown that the magnetic behaviour of $\alpha$ (BEDT-TTF $)_{2} \mathrm{KHg}(\mathrm{SCN})_{4}$ at high magnetic fields has many of the characteristic features of a critical state model, strongly resembling a type II superconductor in a magnetic field [35,36,51]. This critical state-like behaviour, together with the pronounced drop in resistivity at low temperatures, is clearly unlike that expected for a DW systems [34], nor can it be connected with the QHE.

While certain physical aspects of the behaviour of $\alpha$ $(\mathrm{BEDT}-\mathrm{TTF})_{2} \mathrm{KHg}(\mathrm{SCN})_{4}$ at high magnetic fields are similar to those of a type II superconductor, there are, however, many reasons for questioning any interpretation in terms of a field-induced superconducting state. While the presence of a CDW at low magnetic fields indicates that electron-phonon interactions are important in this material, a CDW ground state is invariably always more stable than an electron-phonon mediated superconductor in a strong magnetic field. Both CDW's and singletpaired superconductors are Pauli limited at a critical field $B_{\mathrm{c}}$. However, superconductors have the additional disadvantage of being suppressed by orbital effects at much lower magnetic fields than that at which the Pauli critical field is expected to occur [35]. Thus, if the ground state of $\alpha$-(BEDT-TTF $)_{2} \mathrm{KHg}(\mathrm{SCN})_{4}$ results from the competition between superconductivity and CDW's, as one might gather from the fact that the $M=\mathrm{NH}_{4}$ salt is superconducting and that $\alpha$-(BEDT-TTF $)_{2} \mathrm{KHg}(\mathrm{SCN})_{4}$ becomes superconducting under uniaxial stress, the fact that the CDW has already "won" at $B=0$, makes the emergence of superconductivity in an applied field seem somewhat remote. Furthermore, while several models have been proposed for "reentrant" superconductivity in very strong magnetic fields [51,53 55, a common prerequisite of these models is that the material is already a stable superconductor at $B=0$.

From the CDW perspective, on the other hand, neither the modulated CDW phase nor the CDW-SDW hybrid phase have yet been the subject of detailed theoretical or experimental studies 13,23]. We cannot exclude the possibility that the $\mathrm{CDW}_{x}$ phase itself exists in some form of critical state, which then only mimics the inductive behaviour of a superconductor in strong magnetic fields, and has yet to be identified. With such an explanation, 
however, there would be some difficulty in explaining the pronounced drop in the resistivity at low temperatures in this material. One could also imagine that, within the $\mathrm{CDW}_{x}$ regime, the Frölich CDW mode is no longer efficiently coupled to impurities [34] or to the lattice, leading to dissipationless currents as $T \rightarrow 0$.

Clearly, there exists a challenge in this material, and similar materials within the same series (i.e. the $M=\mathrm{Tl}$ and $\mathrm{Rb}$ salts), to find alternative ways of probing the possible presence of non-dissipative currents (or supercurrents) above $B \sim 24.2 \mathrm{~T}$. The present data indicates only that the high magnetic field phase of $\alpha$-(BEDT$\mathrm{TTF})_{2} \mathrm{KHg}(\mathrm{SCN})_{4}$ exhibits properties that are qualitatively similar to those of type II superconductors in strong magnetic fields. On the other hand, without the existence of a type I superconducting phase, it would be very difficult to experimentally prove the existence of type II superconductivity at such strong magnetic fields. What is clear from these experiments is that the high magnetic field phase of $\alpha$-(BEDT-TTF $)_{2} \mathrm{KHg}(\mathrm{SCN})_{4}$ is very different from any CDW (or SDW) observed in any other material, nor can any of the observations in static magnetic fields be considered consistent with the quantum Hall effect.

\section{ACKNOWLEDGEMENTS}

The work is supported by the Department of Energy, the National Science Foundation (NSF) and the State of Florida. One of us (JSB), acknowledges the provision of an NSF grant (DMR-99-71474), and NH would like to thank Albert Migliori for useful discussions. One of us (LB) would also like to acknowledge the provision of a FSU visiting scientist scolarship. We would futher like to acknowledge Eric Palm and Timothy Muphy for maintaining the dilution refrigerator during the lowest temperature experiments.

[1] T. Ishiguro and K. Yamaji, Organic Superconductors, (Springer, Berlin 1990).

[2] Fermi Surfaces of Low-Dimensional Organic Metals and Superconductors, Springer Tracts in Modern Physics 134, (Springer Verlag, 1996).

[3] For a recent review, see articles in Proceedings of the International Conference on the Science an Technology of Synthetic Metals, Montpellier, France, 1998 [Synth. Met. 103 (1999)].

[4] H. H. Wang, K. D. Carlson, U. Geiser, W. K. Kwok, M. D. Vashon, J. E. Thompson, N. F. Larsen, G. D. McCabe, R. S. Hulscher and J. M. Williams, Physica C 166, 57 (1990).
[5] C. E. Campos, J. S. Brooks, P. J. M. van Bentum, J. A. A. J. Perenboom, S. J. Klepper, P. S. Sandhu, S. Valfells, Y. Tanaka, T. Kinoshita, N. Kinoshita, M. Tokumoto and H. Anzai, Phys. Rev. B 52, R7014 (1995).

[6] T. Hirayama, K. Manabe, H. Akimoto, H. Ishimoto, H. Ito, T. Ishiguro, H. Mori and G. Saito, Czechoslovak J. Phys. 46, 813 (1996).

[7] H. Ito, H. Kaneko, T. Ishiguro, H. Ishimoto, K. Kono, S. Horiuchi, T. Komatsu and G. Saito, Solid State Commun. 85, 1005 (1993).

[8] J. S. Brooks, S. J. Klepper, X. Chen, P. Henning, P. J. C. Signore, M. W. Meisel, N. Kinoshita, M. Tokumoto and H. Anzai, Applied Superconductivty 2, 759 (1994).

[9] H. Ito, M. V. Kartsovnik, H. Ishimoto, K. Kono, H. Mori, N. D. Kushch, G. daito, T. Ishiguro and S. Tanaka, Synth. Metals 70, 899 (1995).

[10] F. L. Pratt, J. Singleton, M. Doporto, A. J. Fisher, T. J. B. M. Janssen, J. A. A. J. Perenboom, M. Kurmoo, W. Hayes and P. Day, Phys. Rev. B 45, 13904 (1992).

[11] M. V. Kartsovnik, A. E. Kovalev and N. D. Kushch, J. Phys. I (France) 3, 1187 (1993).

[12] K. Miyagawa, A. Kawamoto and K. Kanoda, Phys. Rev. B 56, R8487 (1997).

[13] R. H. McKenzie, cond-mat/9706235.

[14] N. Biskup, J. A. A. J. Perenboom, J. S. Brooks, J. S. Qualls, Solid State Commun. 107, 503 (1998).

[15] N. Harrison, Phys. Rev. Lett. 83, 1395 (1999).

[16] N. Harrison, M. M. Honold, M. V. Kartsovnik, J. Singleton, S. T. Hannahs, D. G. Rickel and N. D. Kushch, Phys. Rev. B 55, R16005 (1997).

[17] T. Osada, R. Yagi, A. Kawasumi, N. Miura, M. Oshima and G. Saito, Phys. Rev. B 41, 5428 (1990).

[18] A. A. House, S. J. Bludell, M. M. Honold, J. Singleton, J. A. A. J. Perenboom, W. Hayes, M. Kurmoo and P. Day, J. Phys.: Condens. Matter 8, 8829 (1996).

[19] M. V. Kartsovnik, W. Biberacher, E. Steep, P. Christ, K. Andres, A. G. M. Jansen and H. Müller, Synth. Metals 86, 1933 (1997).

[20] N. Harrison, A. House, M. V. Kartsovnik, A. V. Polisski, J. Singleton, F. Herlach, W. Hayes and N. D. Kushch, Phys. Rev. Lett. 77, 1576 (1996).

[21] S. Hill, P. S. Sandhu, J. S. Qualls, J. S. Brooks, M. Tokumoto, N. Kinoshita, T. Kinoshita and Y. Tanaka, Phys. Rev. B 55, R4891 (1997).

[22] M. M. Honold, N. Harrison, J. Singleton, H. Yaguchi, C. Mielke, D. Rickel, I. Deckers, P. H. P. Reinders, F. Herlach, M. Kurmoo, P. Day, J. Phys.: Condens. Matter 9, L533 (1997).

[23] D. Zanchi, A. Bjelis and G. Montambaux, Phys. Rev. B 53, 1240 (1996).

[24] P. Fulde and R. A. Ferrel, Phys. Rev. 135, A550 (1964).

[25] A. I. Larkin and Yu N. Ovchinnikov, Sov. Phys. JETP 20, 762 (1965).

[26] P. L. Kuhns, J. S. Brooks, T. Caldwell, W. G. Moulton, A. P. Reyes, N. Biskup, A. M. Kini, J. A. Schlueter, H. H. Wang, U. Geiser, J. M. Williams, Solid State Commun. 109, 637 (1999).

[27] M. Tokumoto, N. Kinoshita, H. Anzai, A. G. Swanson, J. S. Brooks, S. T. Hannahs, C. C. Agosta, M. Tamura, 
H. Tajima, H. Kuroda, A. Ugawa and K. Yakushi, Synth. Metals 42, 2459 (1991).

[28] N. Harrison, R. Bogaerts, P. H. P. Reinders, J. Singleton, S. J. Blundell and F. Herlach, Phys. Rev. B 54, 9977 (1996).

[29] A. E. Datars and J. E. Sipe, Phys. Rev. B 51, 4312 (1995).

[30] D. P. Druist, P. J. Turley, K. D. Maranowski, E. G. Gwinn and A. C. Gossard, Phys. Rev. Lett. 80, 365 (1998).

[31] B. Zhang, J. Brooks, Z. Wang, J. Simmons, J. Reno, N. Lumpkin, J. O'Brien and R. Clark, Phys. Rev. B 60, 8743 (1999).

[32] J. T. Chalker and A. Dohmen, Phys. Rev. Lett. 75, 4496 (1995).

[33] L. Balents and M. P. A. Fisher, Phys. Rev. Lett. 76, 2782 (1996).

[34] G. Grüner, Density Waves in Solids (Frontiers in Physics v. 89), (Addison-Wesley Publishing Company, 1994).

[35] M. Tinkham, Introduction to Superconductivity, Second Edition, (McGraw-Hill, 1996).

[36] See e.g. Invited Papers of the Fifth International Conference on Materials and Mechanisms of Superconductivity High Temperature Superconductors, Beijing, 1997 (North-Holland, 1997).

[37] see S. W. Hsu, S. Y. Tsaur and H. C. Ku, Phys. Rev. B 38, 856 (1988) and references included therein.

[38] A. Kovalev, H. Mueller and M. V. Kartsovnik, Sov. Phys. JETP 86, 578 (1998).

[39] T. Sasaki, H. Sato and N. Toyota, Synth. Met. 41-43, 2211 (1991).

[40] F. L. Pratt, T. Sasaki, N. Toyota and K. Nagamine, Phys. Rev. Lett. 74, 3892 (1995).

[41] For a review of all of the data concerning the Fermi surface and its reconstruction, see N. Harrison, E. Rzepniewski, J. Singleton, P. J. Gee, M. M. Honold, P. Day and M. Kurmoo, J. Phys. Condens. Matt. 11, 7227 (1999).

[42] P. Christ, W. Biberacher, W. Bensch, H. Müller and K. Andres, Synth. Metals 86, 2057 (1997).

[43] P. Christ, W. Biberacher, H. Müller, K. Andres, E. Steep and A. G. M. Jansen, Synth. Metals 70, 823 (1995).

[44] P. Christ, W. Biberacher, H. Müller, K. Andres, E. Steep and A. G. M. Jansen, Physica B 204, 153 (1995).

[45] S. Uji, T. Terashima, H. Aoki, J. S. Brooks, M. Tokumoto, N. Kinoshita, T. Kinoshita, Y. Tanaka and H. Anzai, Phys. Rev. B 54, 9332 (1996).

[46] T. Sasaki, H. Sato and N. Toyota, Synth. Met. 41-43, 2211 (1991).

[47] T. Sasaki, A. G. Lebed, T. Fukase and N. Toyota, Phys. Rev. B 54, 12969 (1996).

[48] T. Sasaki and N. Toyota, Phys. Rev. B 48, 11457 (1993).

[49] N. harrison, L. Balicas, J. S. Brooks, J. Sarrao and Z. Fisk, Phys. Rev. B (in press 2000).

[50] C. P. Bean, Phys. Rev. Lett. 8, 250 (1962).

[51] C. A. R. Sá de Melo (Ed.), The Superconducting State in Magntic in High Magnetic Fields, (World Scientific Publishing Co., Singapore, 1998).

[52] M. M. Honold, N. Harrison, J. Singleton, M.-S. Nam, S. J. Blundell, C. H. Mielke, M. V. Kartsovnik and N. D. Kushch, Phys. Rev. B 59, R10417 (1999).
[53] Z. Tesanivic, M. Rasolt and L. Xing, Phys. Rev. Lett. 63, 2425 (1989); M. Rasolt and Z. Tesanovic, Rev. Mod. Phys. 64, 709 (1992).

[54] H. Akera, A. H. MacDonald, S. M. Girvin and M. R. Norman, Phys. Rev. Lett. 67, 2375 (1991).

[55] N. Dupuis, G. Montabaux and C. A. R. Sá de Mela, Phys. Rev. Lett. 70, 2613 (1993).

FIG. $1 . \quad$ The magnetoresistance of $\alpha$-(BEDT-TTF $)_{2} \mathrm{KHg}(\mathrm{SCN})_{4}$ sample 1 at selected temperatures, with the $\mathrm{SdH}$ frequency being $675 \mathrm{~T}$ at $\theta=7^{\circ}$. The "phase-inversion" effect is particularly pronounced in this sample at fields above the kink transition field, with the inverted $\mathrm{SdH}$ minima having resistivity values $\sim 4$ times less that the minimum resistivity at zero field.

FIG. 2. The resistance versus temperature both at integer and half-integer filling factors for two different samples (sample 1 and sample 2) plotted on a logarithmic scale. In both samples, the resistance varies weakly with temperature until it drops abruptly at $\sim 3 \mathrm{~K}$, falling in an exponential fashion. While the effect is more abrupt at integer filling factors, a sharp kink is also observed at half integer filling factors.

FIG. 3. An example of the magnetic torque measured in sample 3 at the same orientation $\left(7^{\circ}\right)$ as that of the transport meaurements made on sample 1 , at $500 \mathrm{mK}$, versus magnetic field squared. A quadratic fit has been made to the torque at fields below $15 \mathrm{~T}$. Rather than plotting $\tau$, we have plotted $-\tau$, so that the sign of the magnetic torque is the same as that of $\chi_{\perp}$.

FIG. 4. Field-cooled magnetic torque measurements made at various different values of magnetic field, indicating that the change in slope continues to be discernable even at the highest available static magnetic fields. The torque has been normalised by $1 / B$.

FIG. 5. The magnetic torque measured for sample 3 in rising and falling magnetic fields between 15 and $32 \mathrm{~T}$. The lower (higher) of the two curves corresponds to the up (down) sweep, as indicated by the lower (upper) arrow. The temperature of the dilution refrigerator was $27 \mathrm{mK}$.

FIG. 6. (a) An example of a hysteresis loop observed in $\alpha$-(BEDT-TTF $)_{2} \mathrm{KHg}(\mathrm{SCN})_{4}$ as a result of sweeping the magnetic field back and forth several times over the same interval at different rates between $8 \mathrm{mTs}^{-1}$ and $50 \mathrm{mTs}^{-1}$. Part of the hysteresis obtained by sweeping the magnetic field over the entire range between 24.4 and $32 \mathrm{~T}$ is also shown. (b) The same hysteresis after subtracting the dHvA and monotonic background magnetic torque. 
FIG. 7. Relaxation of the magnetic torque observed at an integer filling factor (a) and half-integer filling factor (b), with time $t$ plotted on a logarithmic scale. At half-integer filling factors, the moment appears to decay logarithmically, as expected for a type II superconductor.

FIG. 8 (a) The hysteresis observed in $\alpha$-(BEDT-TTF $)_{2} \mathrm{KHg}(\mathrm{SCN})_{4}$, measured in the dilution refrigerator at several different temperatures, obtained by subtracting the down sweep data from the up sweep. The waveform of the hysteresis is complicated, being largest at half-integer filling factors at higher temperatures, then becoming more pronounced at integer filling factors at the lowest temperature. (b) The dHvA signal extracted from the same data by summing up and down sweeps. The monotonic background magnetic torque has been subtracted for clarity.

FIG. 9. A logarithmic plot of the maxima in the hysteresis versus $T$ at half-integer filling factors $(B \sim 30.1 \mathrm{~T})$ and integer filling factors $(B \sim 30.7 \mathrm{~T})$.

FIG. $\quad 10 . \quad$ A tentative phase diagram for $\alpha$-(BEDT-TTF $)_{2} \mathrm{KHg}(\mathrm{SCN})_{4}$ in a magnetic field, for small values of $\theta$. The points have been extracted both from magnetotransport and magnetic torque measurements discussed in this work, as well as specific heat measurements of Kovalev et al.. 
figure 1 of Harrison et al

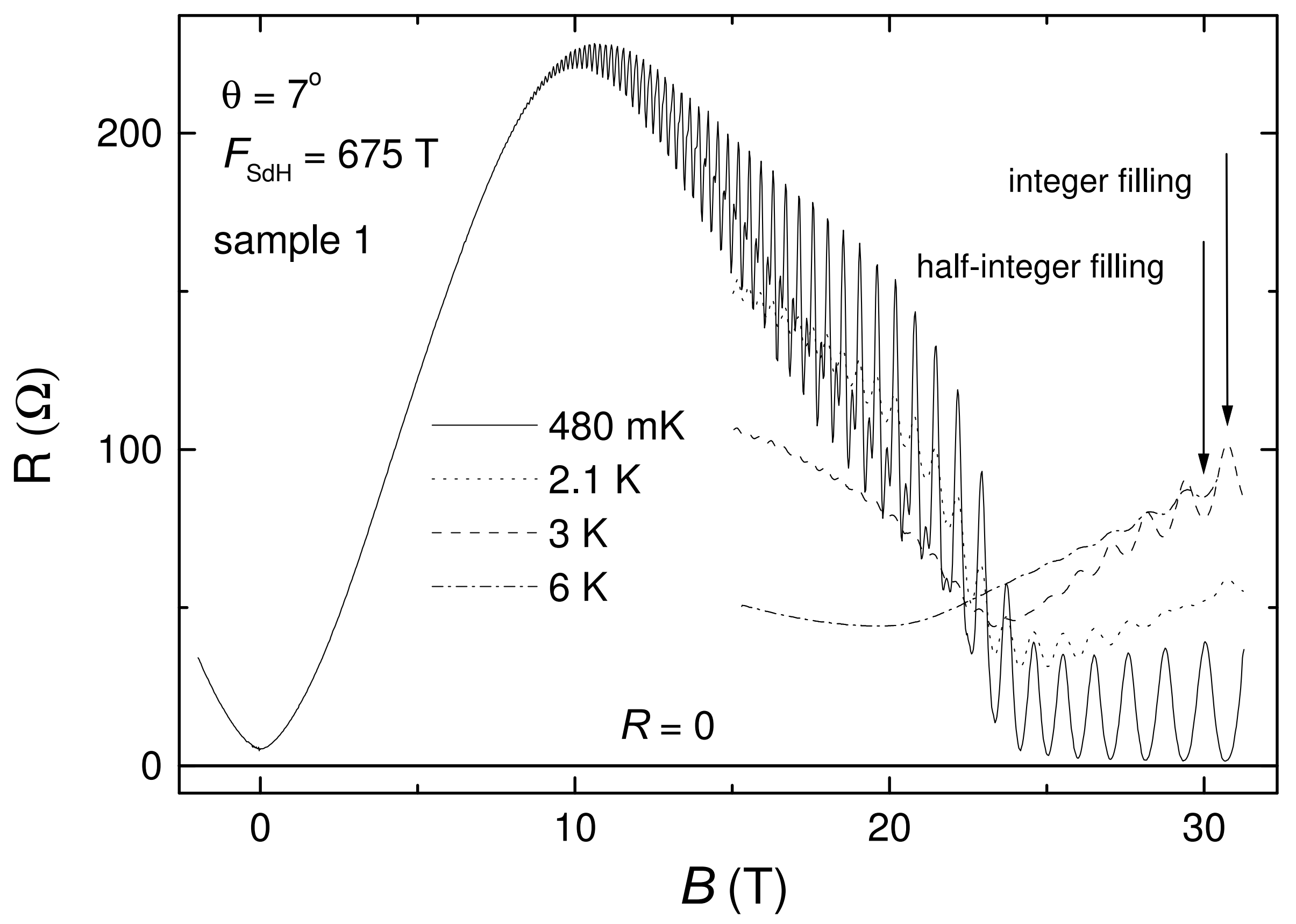


figure 2 of Harrison et al
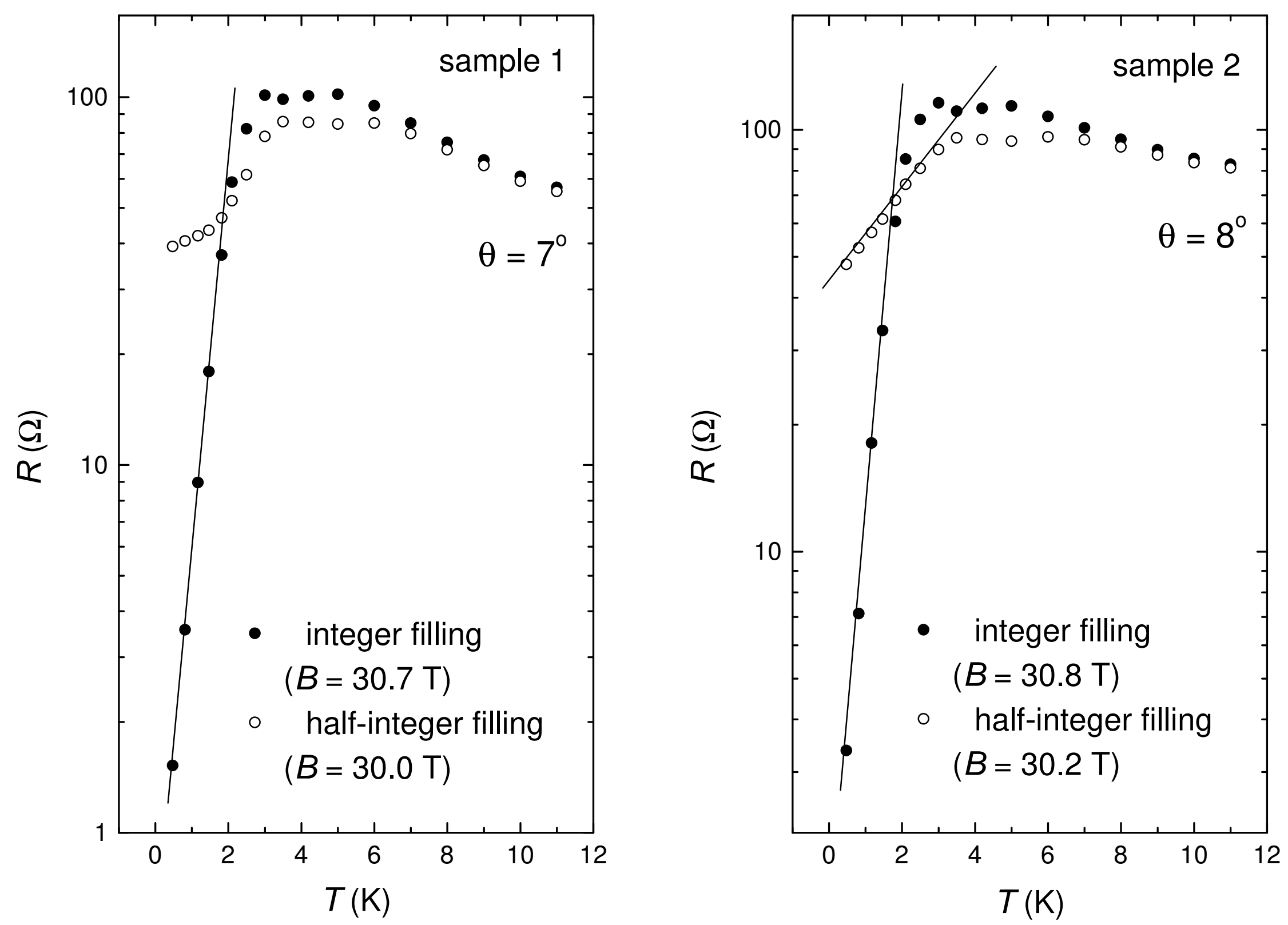
figure 3 of Harrison et al

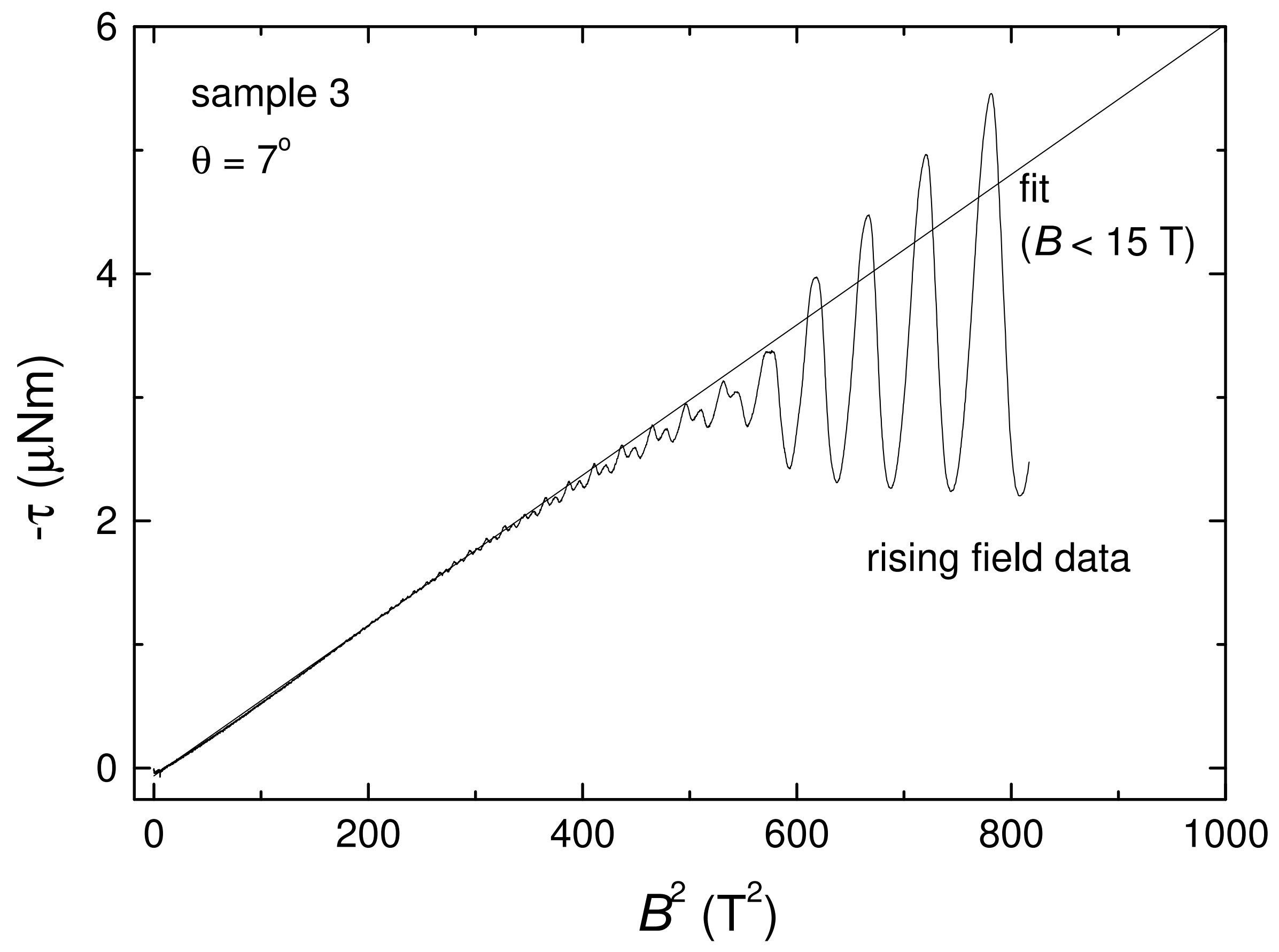


figure 4 of Harrison et al

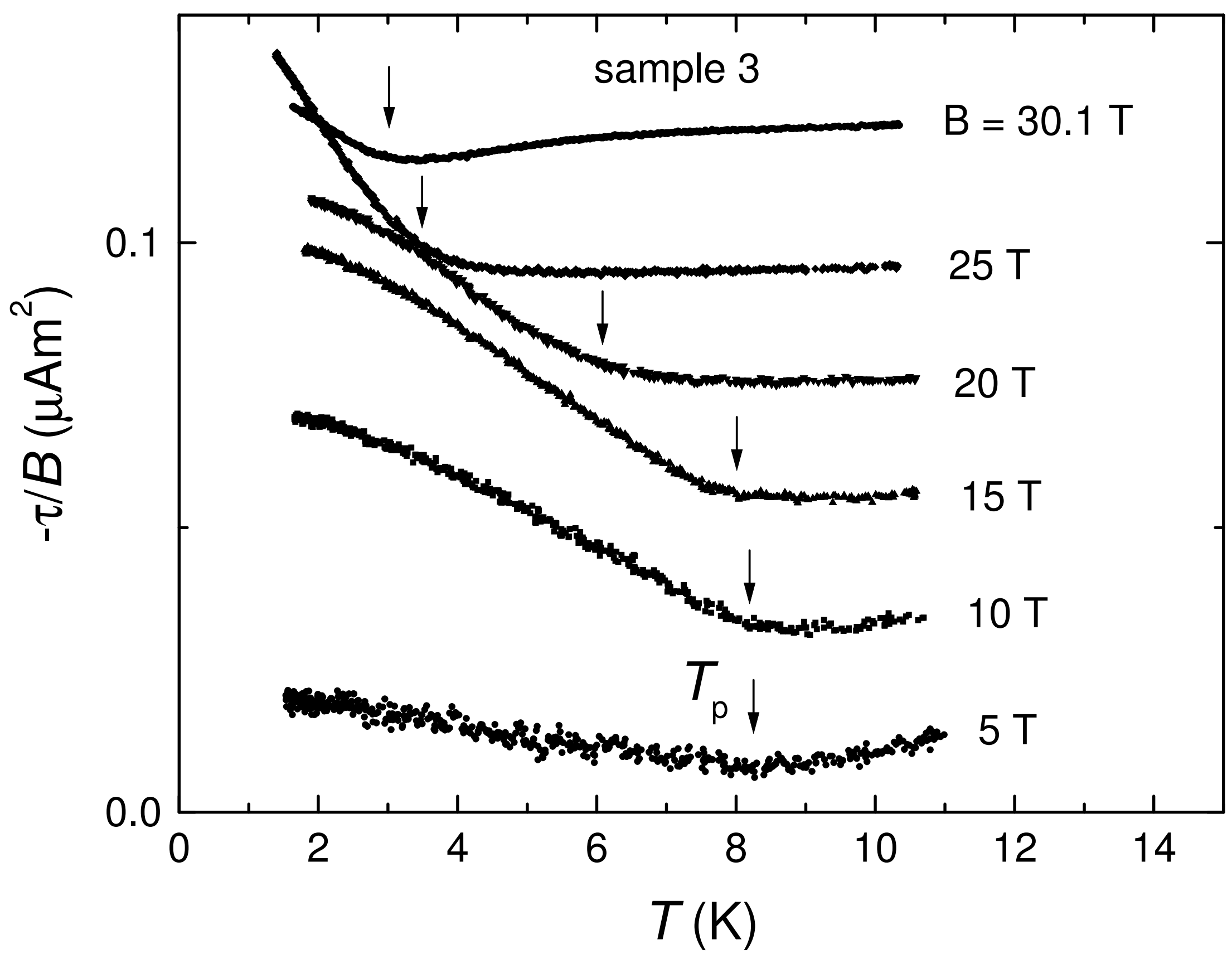


figure 5 of Harrison et al

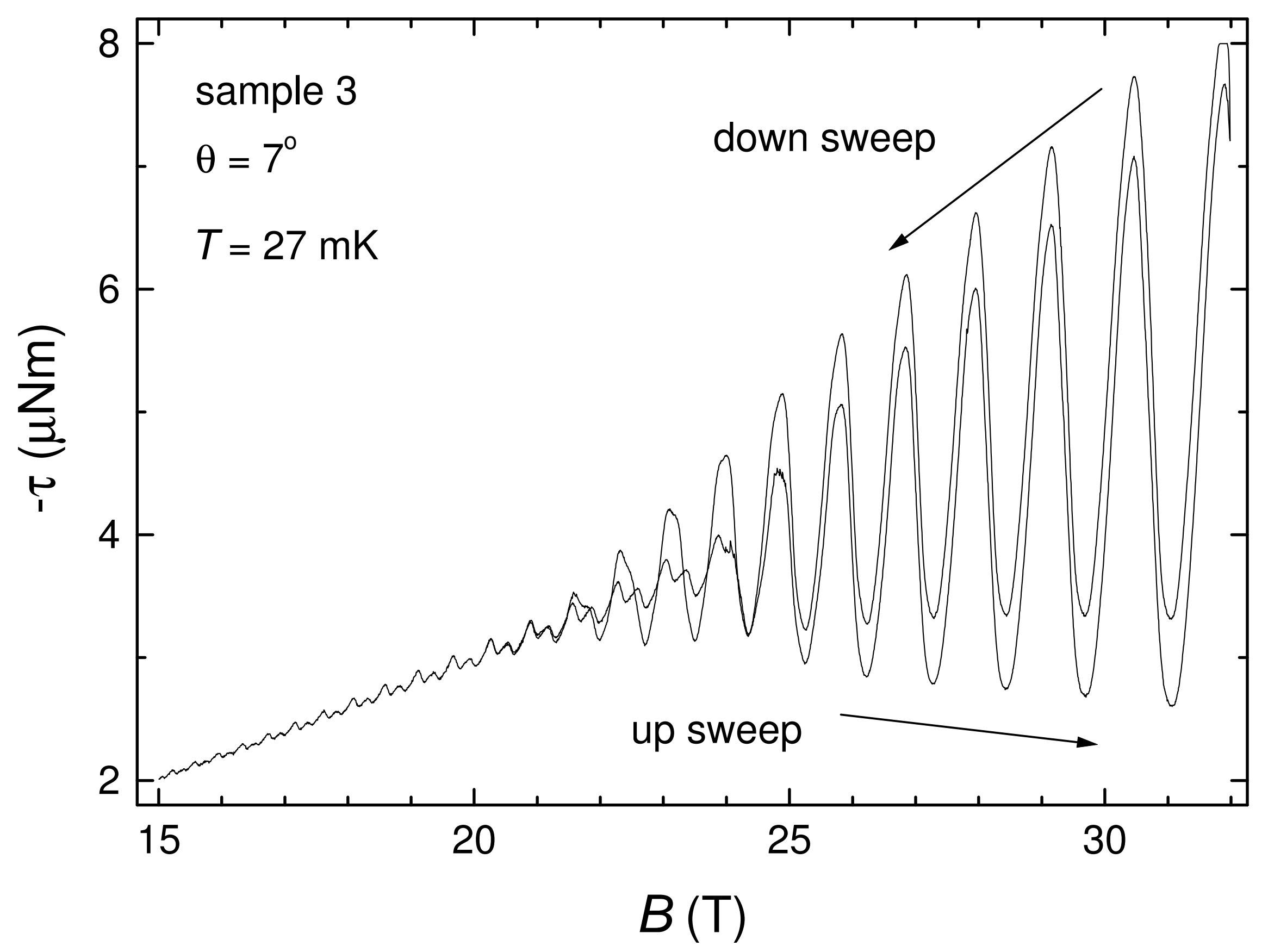


figure 6 of Harrison et al
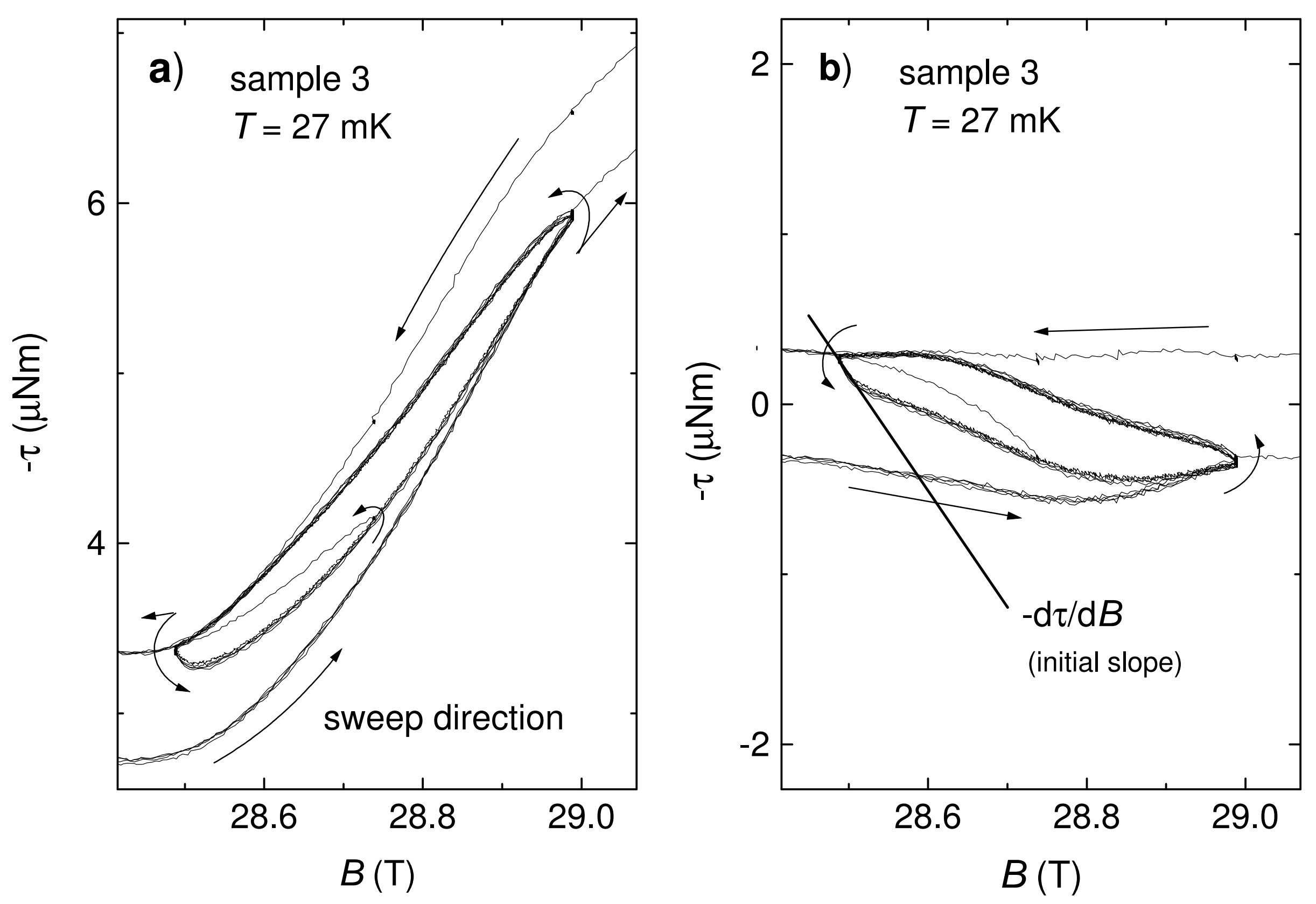
figure 7 of Harrison et al
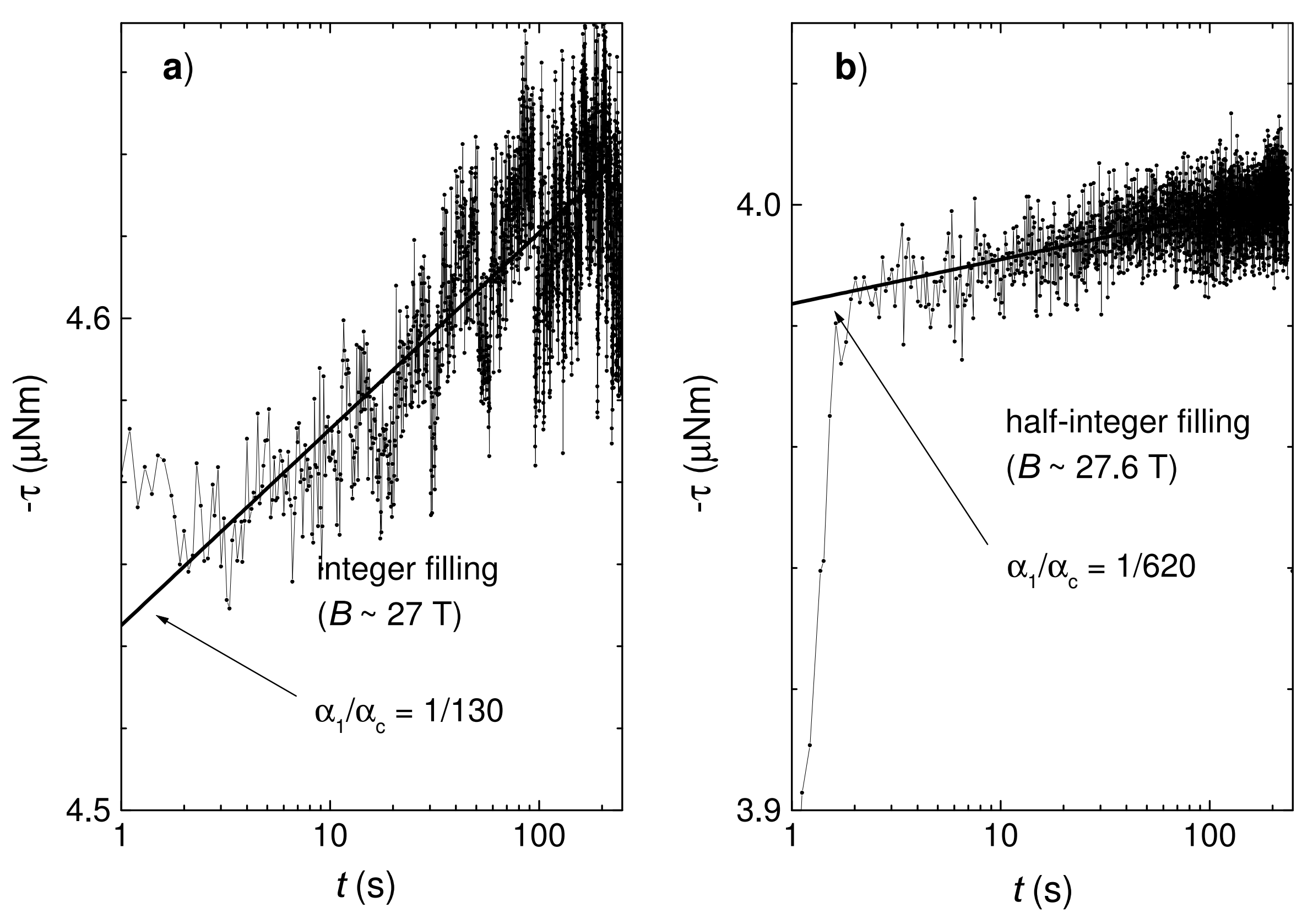
$B(\mathrm{~T})$

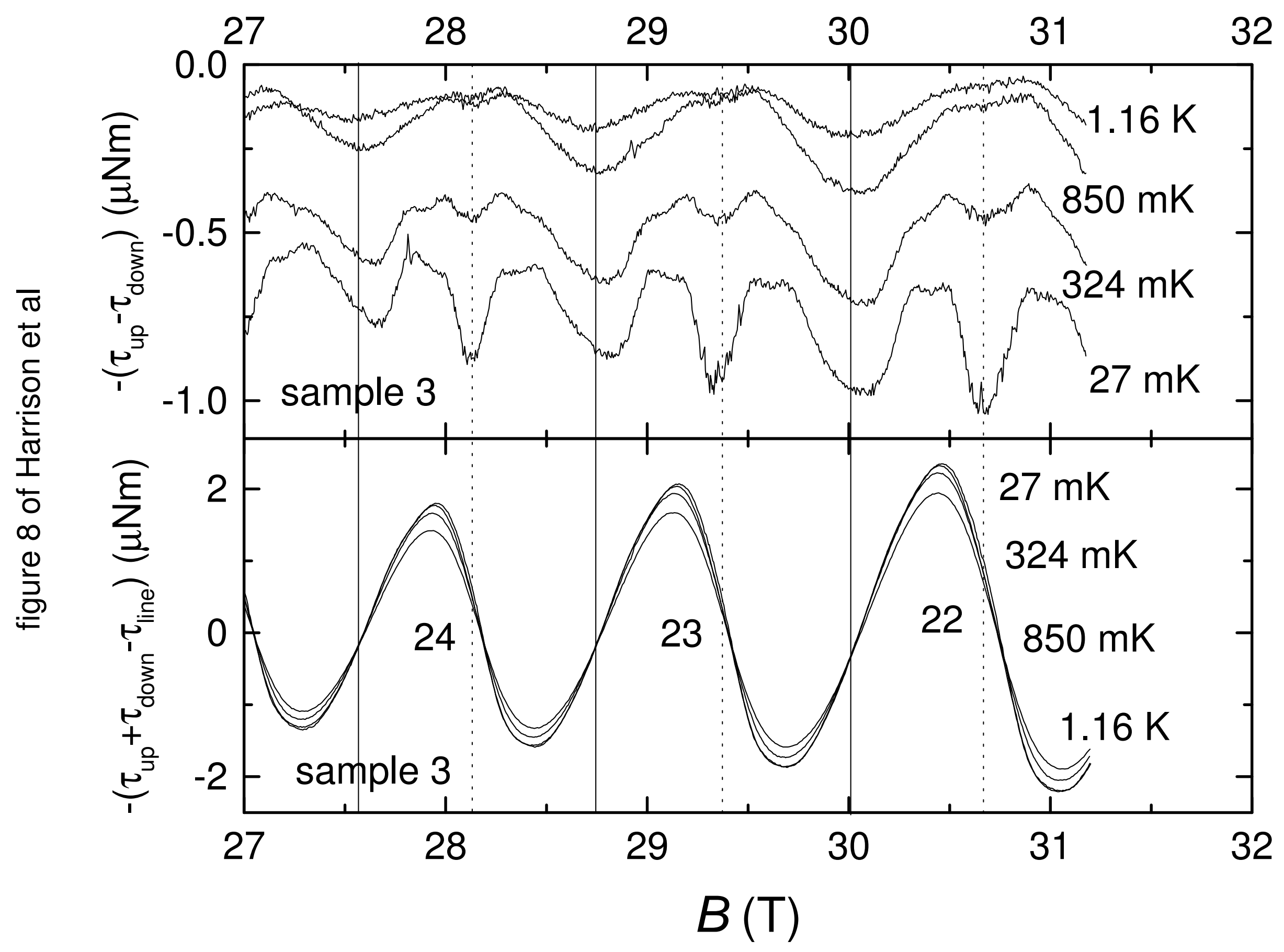


figure 9 of Harrison et al

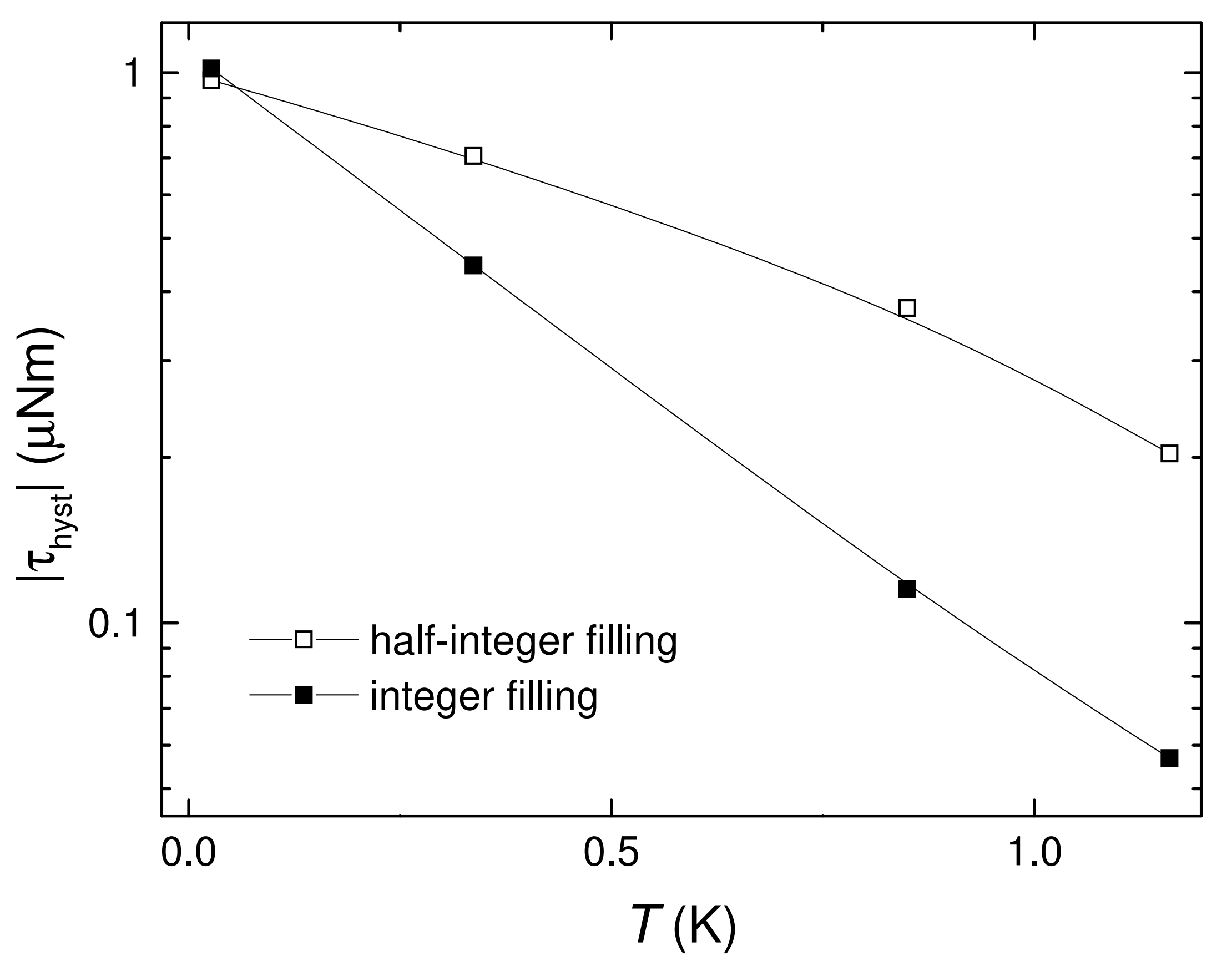


figure 10 of Harrison et al

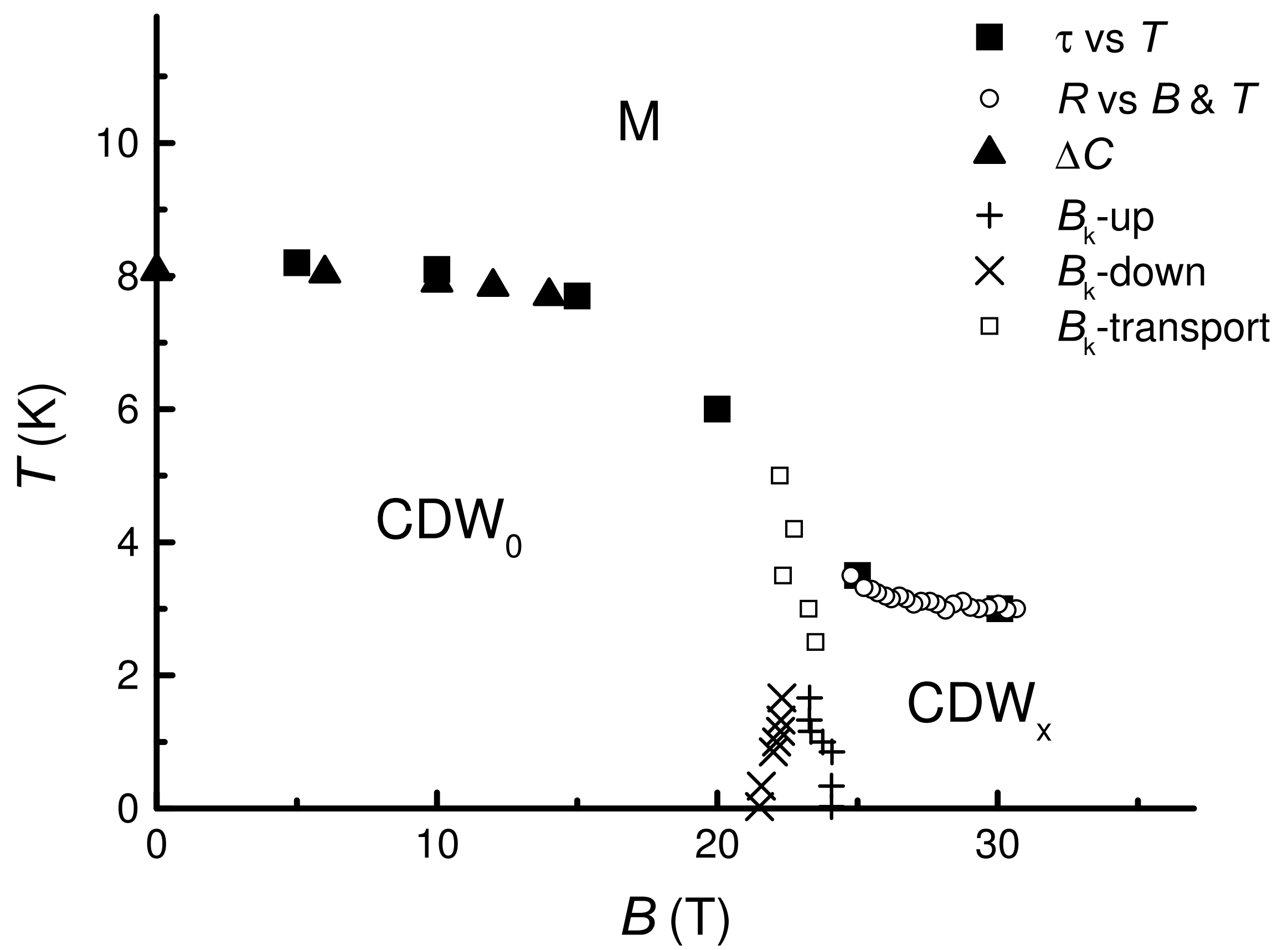

